\title{
Experiences of Occupational Stress among Emergency Nurses at Private Hospitals in Bangkok, Thailand
}

\author{
Nuttapol Yuwanich1 ${ }^{*}$, Sharareh Akhavan1, Walaiporn Nantsupawat², Lene Martin ${ }^{1,3}$ \\ ${ }^{1}$ School of Health, Care and Social Welfare, Mälardalen University, Eskilstuna and Västerås, Sweden \\ ${ }^{2}$ School of Nursing Science, Rangsit University, Pathumthani, Thailand \\ ${ }^{3}$ School of Health Sciences, University of London, London, UK \\ Email: ${ }^{\star}$ nuttapol.yuwanich@mdh.se, ${ }^{\star}$ nuttapol.yuwanich@gmail.com, sharareh.akhavan@mdh.se, walaiporn.nan@rsu.ac.th, \\ lene.martin@mdh.se
}

How to cite this paper: Yuwanich, N., Akhavan, S., Nantsupawat, W. and Martin, L. (2017) Experiences of Occupational Stress among Emergency Nurses at Private Hospitals in Bangkok, Thailand. Open Journal of Nursing, 7, 657-670.

https://doi.org/10.4236/ojn.2017.76049

Received: May 9, 2017

Accepted: June 20, 2017

Published: June 23, 2017

Copyright $(9) 2017$ by authors and Scientific Research Publishing Inc. This work is licensed under the Creative Commons Attribution International License (CC BY 4.0).

http://creativecommons.org/licenses/by/4.0/

\begin{abstract}
Introduction: Occupational stress has negative effects on employee's health and organizational productivity. Nurses in emergency department are more exposed to stress than nurses in other departments. Aim: To explore nurses' experiences of occupational stress in emergency departments in private hospitals in Bangkok, Thailand. Design: A descriptive qualitative design, with a deductive approach based on the Job Demand-Control-Support model was used. Methods: Fifteen emergency department nurses at two different hospitals were interviewed and the data were analyzed using a manifest content analysis. Results: Three main categories: "work context is an issue", "consequences of reactions to stress", and "coping with work stress", including seven sub-categories emerged from the data analysis. Conclusion: The patients' and their relatives' behaviors were experienced as the primary stressor at the private hospital, in addition to excessive work tasks. Other important stressors were misunderstanding and conflicts between emergency department nurses and the other health care professionals, presumably related to hierarchy and power relations between health care professions. Creating a better working environment and a balance between the number of patients and nurses would reduce workload and stress, encourage ED nurses to stay in the profession and ultimately maintain patient safety.
\end{abstract}

\section{Keywords}

Emergency Department, Emergency Room Nursing, Occupational Stress, Private Hospitals

\section{Introduction}

Nursing is considered a stressful occupation, particularly in emergency depart- 
ments (ED), where nurses encounter unpredictable events and specific stressors, such as trauma, violence, acute life-threatening conditions, sudden death and overcrowding [1]. Furthermore, they report more time pressure, higher job demands, lower decision-making authority, fewer adequate work procedures and fewer rewards compared to general hospital nurses [2]. ED nurses in public hospitals in Thailand have reported problems with heavy workloads, stressing situations involving patients and their relatives, violence in the ED, lack of skill improvement, low income, and difficult relationships within the nursing team. These problems affected the ED nurses' physical and mental health, family relationships, job satisfaction, and the quality of their nursing care [3]. Emergency care is also provided in the private care sector, but less is known if the experience of stress among ED nurses is the same as in public care sector. Therefore, the current study focuses on ED nurses' experiences of occupational stress in private hospitals in Thailand.

\section{Background}

Karasek and Theorell [4] defined occupational stress using the Job DemandControl-Support (JDCS) model, which describes interactions between demands, control and support at work. Job demand refers to any psychological demand at work such as workload or time pressure. Job control dimension is divided into two aspects including skill discretion, which refers to the opportunity of skill used by the employee in his or her workplace, and decision authority refers to the autonomy of an employee to make a task-related decision by themselves at work. The support dimension refers to the overall support at work that is available for a worker [4]. According to the model, employees who experience high psychological demands with low level of job control and low social support are likely to have a greater risk of stress and to suffer from poor health [4]. Occupational stress occurs when job requirements mismatch workers' capabilities, resources, or needs. It can cause a variety of psychophysiological health-related problems and may have important consequences for the organization [5].

Previous studies have shown that occupational stress leads to negative consequences for ED nurses, such as stress-related psychophysiological illnesses, increased arousal and feeling of uneasiness [1] [3] [6] [7]. Due to this, ED nurses have higher rates of absenteeism and sick leave, decreased work performance [2] [8], more work-home conflicts and more intentions to leave the profession compared with nurses who work in other environments [2]. These effects relate to both ED nurses' considerable psychological job demands and to a perceived lack of supervisor support [8]. Ultimately, the consequence may be impaired patient care [2].

The current study focuses on ED nurses working in private care in Thailand. The majority (89.5\%) of the registered nurses (RN) in Thailand work in the public sector [9], where the care is funded by the government. In contrast, private hospitals require patient payment, usually through self-pay, private health insurance or employer-provided health insurance, but occasionally also through 
the Social Security System [10]. Differences in patient populations between public and private hospitals have been described; people with low-income use public and people with middle- to high-income tend to use private hospitals [11]. Due to economic expansion, globalization, international trade, and medical hub policies in Thailand [12] the number of private hospitals have increased [10] [13]. They host primary, secondary and tertiary care under the same roof and provide specialized care and treatment, including high-technology surgery. The services aim to meet all the patients' needs and expectations, but the payment system favors patients with high incomes [14]. Public and private hospitals should provide the same standard of nursing care for all patients [15]. Nursing practice in Thailand is also influenced by Thai culture and beliefs, especially from Buddhist perspectives [16]. In relation to nursing, the "kreng jai" concept implies that junior nurses have to pay respect to both senior nurses and physicians [16]. This can, for example result in that junior nurses may have to carry out tasks that senior nurse or physicians require them to do, even if they do not feel comfortable with it, which may influence their decisions regarding nursing care.

Tyson and Pongruengphant [17] studied stress in nurses in both the public and the private sector. They found that high workload, involvement in circumstances concerning life and death, interruptions of nurses' work by management, and performing tasks considered physicians' duty, caused stress among nurses in private hospitals. Raungsrijan and Suppapitiporn [18] showed that $63 \%$ of the nurses in a private hospital had high levels of stress, which was associated with disrespect from patients, colleagues and supervisors. Most studies in this area are quantitative and few qualitative studies have specific focus on ED nurses' experiences of stress in private hospital. Therefore, the aim of the current study is to get a deeper understanding of nurses' experiences of occupational stress in EDs in private hospitals in Bangkok, Thailand.

\section{Methods}

\subsection{Study Design}

A descriptive qualitative design, with a deductive approach based on the JDCS model was used.

\subsection{Setting and Participants}

Semi-structured interviews were conducted between May and August 2013. Twenty-eight ED nurses from two large private hospitals were recruited. The hospitals have more than 200 inpatient beds each and provide a variety of treatments and health care services. Therefore, the participants were likely to have relevant experience in emergency care and a broad perspective of stressful situations. The inclusion criteria were RNs of either gender who worked full-time and had at least one year of experience in emergency care. RNs with less than one year of experience in emergency care or were working part-time were excluded. All ED nurses working at the two hospitals $(n=28)$ were invited to participate; 11 were excluded because they had less than one year of experience in 
emergency care, and two ED nurses were not willing to participate. All had a bachelor degree in nursing science and worked at a nurse practitioner level. The demographic information of the participants is shown in Table 1.

\subsection{Data Collection}

The interview guide (Table 2) was based on previous studies [1] [3] [19]. Some interview guide questions were inspired by the JDCS model [4], for example "Could you tell me about your job description and your responsibility in your position?", related to job demand, and "Do you think you have control over your work tasks? Work situation? Working hours?”, related to job control. During the interview (performed by the first author), follow-up questions were used to obtain a better understanding of the participants' experiences. The interviews were performed during the nurse's work hours (morning, evening, or night shift). Each participant was interviewed in a private room in the ED or in a private location of his or her choice. The interviews lasted until data saturation was obtained, between 25 and 80 minutes.

\subsection{Data Analysis}

The audio-recorded interviews were transcribed verbatim by the first author (NY) and translated into English. The transcriptions were reviewed by a professional linguist to confirm their accuracy, consistency and to determine conceptual equivalence. A content analysis [20] with a deductive approach based on the

Table 1. Description of the participants demographics (age, gender, job experience and marital status).

\begin{tabular}{cc}
\hline Demographic information & $(\mathbf{n}=15)$ \\
\hline Age; Md (range) & $27(20-39)$ years \\
Gender; f/m & $12 / 3$ \\
Emergency care experience; range & $1-10$ years \\
Marital status; single/married & $12 / 3$ \\
\hline
\end{tabular}

$\mathrm{f}=$ female, $\mathrm{m}=$ male, $\mathrm{Md}=$ median, $\mathrm{n}=$ number .

Table 2. Interview guide, including 7 questions and examples of follow-up questions that could be asked to get a better understanding of the respondents' answers.

1. Could you tell me about your job description and your responsibility in your position?

2. Do you think you have control over your work tasks? Work situation? Working hours?

3. Can you choose your work tasks? How?

4. Could you tell me what is stressful in your workplace according to your perception?

5. Would you please describe why these factors cause stress in your workplace?

6. Does the stress in your workplace influence your health? In what way does it affect your health?

7. Does your workplace offer you resources/possibilities/facilities to cope with work stress? If yes, what? If no, why?

Follow-up questions:

Could you describe that experience?

How do you feel about this situation?

How does this situation makes you feel stress? 
JDCS model was used to conceptually describe the nurses' experiences of occupational stress. The analysis procedure is depicted in Figure 1.

\subsection{Ethical Approval}

The study was approved by the Regional Ethical Review Board in Uppsala, Sweden (Dnr. 2013/087) and the research ethics committees of the included hospitals in Thailand. The study was conducted in accordance with the Helsinki Declaration. Written consent was obtained before the interviews began.

\section{Results}

Three main categories, including seven sub-categories, emerged from the data analysis and are shown in Figure 2.

\subsection{Work Context Is an Issue}

This category described what the participants experienced as causes of stress in the EDs in private hospitals. The main causes were related to the participants' workplace context, and described by three sub-categories.

\subsubsection{Encountering the Excessive Work Tasks and the Nature of ED Work Characteristics}

The work characteristics of the ED were sources of stress. Providing urgent nursing care to critically ill patients implies working under pressure and in unpredictable situations. Multiple work tasks and excessive work hours, and in particular, tasks beyond the nursing role made them experience a high level of
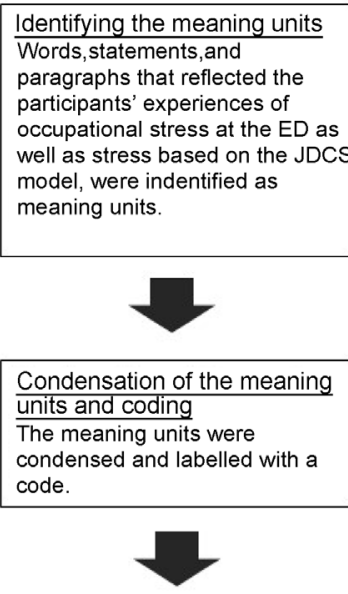

Creating the sub-categories and categories

The various codes were

compared due to differences and similarities, and they were then sorted into sub-categories.

Finally, the emerged subcategories that had a similar meaning were categorized into a category which constitude the manifest content.

\begin{tabular}{|c|c|c|}
\hline & Interview\#1 & Interview\#2 \\
\hline Meaning units & $\begin{array}{l}\text { You have to understand that patient's } \\
\text { relatives at the private hospital just } \\
\text { want to talk with the physician, not the } \\
\text { nurses. They do not trust when they } \\
\text { talk to the nurses. We have inadequate } \\
\text { physicians here;thus, talking to the } \\
\text { patient's relatives becomes our } \\
\text { responsibility. }\end{array}$ & $\begin{array}{l}\text { There is a problem associated with the ability for patients to pay at a } \\
\text { private hospital. Iwould say this is totally different from a public } \\
\text { hospital. In cases of accidents and emergencies, when the patient has } \\
\text { been transferred to ER, we have to help them first because our } \\
\text { department is different from others, such as the medical-surgical } \\
\text { outpatient department or the health check-up unit, where patients could } \\
\text { make a plan and independently choose to have health care services by } \\
\text { themselves. Whenever we could not clear a patient's finances or could } \\
\text { not find a hospital to refer the patient to for further treatment due to their } \\
\text { right-of-health care scheme, this makes me feel stressed, because it is } \\
\text { something that I cannot control and I have to ask the nurse supervis or } \\
\text { for help }\end{array}$ \\
\hline $\begin{array}{l}\text { Condensed } \\
\text { meaning untis }\end{array}$ & $\begin{array}{l}\text { A patient's relative at the private } \\
\text { hospital wants to talk to the } \\
\text { physician, not to nurses. They do } \\
\text { not trust the nurses. We have } \\
\text { inadequate physicians here; thus, } \\
\text { talking to the patient's relatives } \\
\text { becomes our responsibility. }\end{array}$ & $\begin{array}{l}\text { In cases of accidents and emergencies, when the patient has been } \\
\text { transferred to ER, we have to help them first because our } \\
\text { department is different from other. When we cannot clear a } \\
\text { patient's finances or find a hospital to refer the patient for further } \\
\text { treatment, this makes me feel stressed and need help from the } \\
\text { nurse supervisor. }\end{array}$ \\
\hline Codes & $\begin{array}{l}\text { Patient's relative do not trust the } \\
\text { nurses. }\end{array}$ & The patient's ability of paying for emergency care services. \\
\hline Sub-categories & $\begin{array}{l}\text { Confronting the situation of } \\
\text { providing emergency care for } \\
\text { patients and their relatives. }\end{array}$ & $\begin{array}{l}\text { Confronting the situation of providing emergency care for patients } \\
\text { and their relatives. }\end{array}$ \\
\hline Category & & Nork context is an issue. \\
\hline
\end{tabular}

Figure 1. Example of the analysis process from identifying to condensation of meaning units to finally creating sub-categories and categories. 


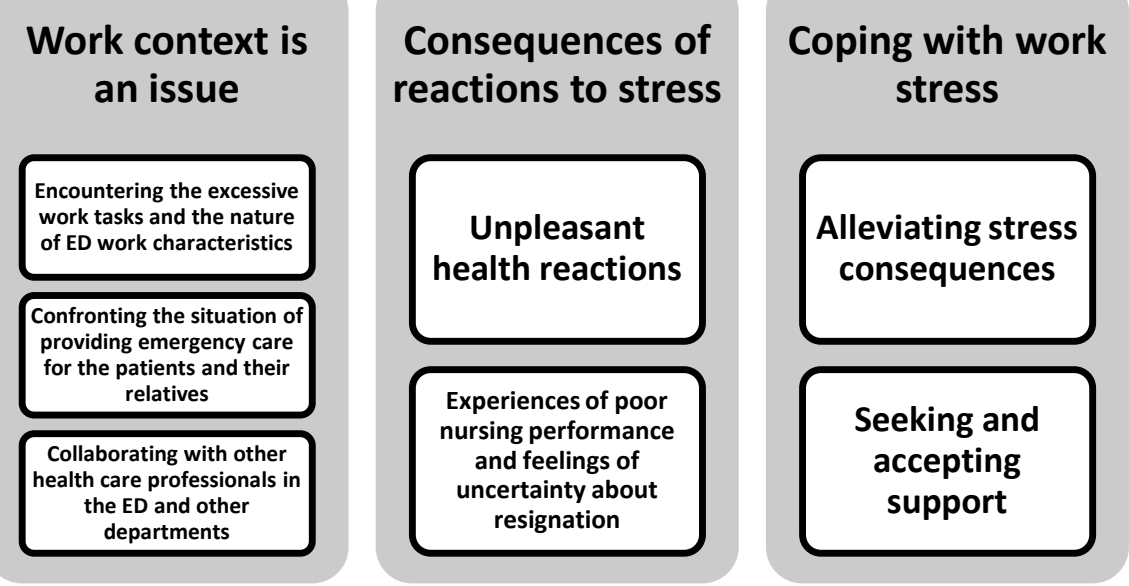

Figure 2. The result showing the three categories and seven sub-categories that emerged from the analysis.

stress. They described having to perform physicians' tasks while waiting for the physician to arrive at the ED and perceived these circumstances as uncontrollable situations at work: "If we keep waiting for them (physicians), the patient might get worse. We are on the front line; we must do something, inevitably" [RN5]. The senior ED nurses had to concurrently perform several tasks; simultaneously caring for patients assigned to junior nurses and their own patients. One senior ED nurse stated:

I have to take care of them (newly graduated nurses), as a mentor ... It is like double tasks at the same time because I have to take care of my assigned patient, the newly graduated nurses, and their assigned patients! This make me stressed because of too many tasks at the same time [RN5].

Lack of work experience was also associated with stress. The participants who had only worked 1 - 2 years in the ED experienced problems with work management and decision-making. When they were in charge, they felt that they could not manage both their work tasks and their colleagues, particularly the senior nurses. A high number of patients and a shortage of nursing staff also increased workload and stress: "They (hospital directors) reduced hiring of new staff. This leads to shortage of nursing staff, and sometime there is a high workload, too many patients and not enough nursing staff. This makes us stressed" [RN7].

\subsubsection{Confronting the Situation of Providing Emergency Care for the Patients and Their Relatives}

The participants described the situations of providing emergency care for patients and their relatives as the most common stressor including unpredictable patient conditions, waiting time for treatment, and the patient/relatives misunderstanding of the triage system. The medical service at an ED relies on the priority of patients with more severe conditions; however, the participants described that some patients and their relatives did not understand that and felt 
that they should be treated with the same priority as patients with severe conditions:

A patient came to the ED with a common cold, but another patient came with a severe cut. The one who had the common cold did not understand and demanded to be treated first. He/she did not listen to us and perceived that he/she had the right to be treated the same as the patient with the severe condition [RN7].

Private hospital patients and their relatives also had high demands and expectations because they paid a high fee for medical and nursing services. Sometimes they behaved disrespectfully, which had a detrimental effect on the participants. Moreover, the participants explained that in Thai culture, people value physician more highly than nurses and feel like nurses are not health care professionals:

They (patients and their relatives) just want to talk to the physician, not with the nurse because they do not believe and respect us as a health care professional. As you know, in Thai culture, the people give a high value to a physician, not a nurse like us [RN3].

The participants described that the ED differs from other healthcare facilities, such as the medical-surgical outpatient department, where patients can book an appointment with the physician when they are in need of care and treatment. In contrast, when someone has an accident and arrives at the ED, hospital policy requires the ED staff to provide health care whether the patient can pay or not. The participants indicated that this situation was a cause of stress:

In cases of accidents and emergencies, when the patient has been transferred to ED, we have to help them first because our department is different from others... When we cannot manage a patient's finances issue or find a hospital to refer the patient for further treatment... this makes me feel stressed [RN9].

\subsubsection{Collaborating with Other Health Care Professionals in the ED and other Departments}

Working with other health care professionals in the ED and other departments was expressed as a stressor and contributed to conflicts among ED nurses, nurses in other departments and other health care professionals, such as pharmacists and particularly physicians. Some physicians rarely provided treatment according to the hospital treatment guidelines, which led to conflicts between the ED nurses and the physicians: "They (physicians) do not follow the treatment flow or guideline of the hospital's treatment standard. In this case, I have no power to address them because I am just a nurse in a practitioner level" [RN9]. Such conflicts created misunderstandings and miscommunication among ED nurses and other health care professionals. They also depended on the hierarchy and the differences in power relations among health care professions. One junior ED nurse described her experience: "It is like a colleague who is older than me, such as nurse aid or practical nurse sometimes, I ask them to 
do something, but they do not listen and do not do what I ask them for" [RN2].

The ward nurses, especially the intensive care unit (ICU) nurses, often refused to receive patients from the ED or demanded the ED nurses to perform all of the nursing procedures for patients before the ICU accepted them. This resulted in conflicts between ward nurses and ED nurses: "They (ICU nurses) are rather fastidious when we have to transfer patients to them. They ask so many questions, and we have to do everything completely before transferring a patient to them. So this makes me feel stressed" [RN10].

The participants described conflicts among the emergency nursing team, particularly personal conflicts related to people's particular behaviors. These conflicts led to an inability for the nurses to work together: "It is like I am in the team that... let's say it simply: we do not get along well. We cannot work together, just work independently" [RN11].

\subsection{Consequences of Reactions to Stress}

This category reflected the ED nurses' reactions to stress consequences, mainly related to ill health and responses to their work at the ED.

\subsubsection{Unpleasant Health Reactions}

Stress in the ED commonly triggered psychophysiological ill health. One ED nurse described her experiences with poor physical health experiences: "I get headaches when I feel stressed; it is so tense, and I feel very uncomfortable! And also stomachaches, as always ... Yes, when I feel stressed, I get them all the time" [RN14]. Another ED nurse shared her experiences of poor psychological health caused by occupational stress: "Stress makes me feel unhappy at work. I feel frustrated, and then I keep quiet and do not want to talk to anyone" [RN6]. The nurses also reported social problems when they encountered stress at work. This problems mainly affected relationships with their family and partner: "Sometimes I have arguments with some of my family members or my boyfriend" [RN2].

\subsubsection{Experiences of Poor Nursing Performance and Feelings of Uncertainty about Resignation}

Stress in the ED contributed to feelings of uncertainty about whether to leave or continue working at the current workplace among the ED nurses: "I thought that it would be nicer to work in a private hospital than in a public hospital, but now I want to resign from here and work in a public hospital instead. I have stress here" [RN2]. Furthermore, the ED nurses described their experiences that stress affected the delivery of nursing care. They provided incomplete nursing care, and they worked more slowly because of stress: "Stress affects my nursing care. I may forget to do something to complete the physician's order... I may forget to send the blood specimen to the laboratory. There are errors sometimes, I would say" [RN4].

\subsection{Coping with Work Stress}

This category described the ED nurses' attempts to cope with work stress and 
contained two sub-categories: alleviating stress consequences, and seeking and accepting support.

\subsubsection{Alleviating Stress Consequences}

The ED nurses stated three types of coping behaviors including avoidance, debriefing, and positive reframing. Some described that when they were on duty and experienced stress, they walked away from the situation for a while and returned when they felt better:

If I feel stressed while I am working, I will take a break, walk away, and then I will come back to fix the problem. The reason that I walk away is so I can plan and think about how to solve the problem [RN3].

In the workplace, debriefing by talking with colleagues, especially close and trusted ones, was an effective method of stress release among the emergency nursing team: "I do have a talk with colleagues when I feel stressed, and it helps a lot. Especially the closest one because it is like we feel the same" [RN11].

They also alleviated stress while they were at work by turning stress into a challenge and thinking positively: "I fight it (stress) by smiling (smiles). Sometimes I like it. They (the patients) ask me many questions, and I give them a lot of information as well. It is like a challenge" [RN4]. Additionally, they described several stress-releasing methods in daily life, such as sleeping, exercise, and making merits and meditating according to Buddhist practice. The effectiveness of these coping behaviors depended on the individual's conception of life.

\subsubsection{Seeking and Accepting Support}

The organization provided some support for reducing employee stress, for example, by providing sports activities: "There is no stress-relief program or anything like that at this hospital. The hospital director just gives us a stress assessment survey. Whoever gets a high stress score is sent to consult with the psychiatrist" [RN4]. The greatest support described came from colleagues, head nurses, and nurse supervisors. That support consisted primarily of debriefing and discussing the problem at work, which relieved the participants' stress and enabled them to continue working. Although they described receiving some support from their department and organization, the participants noted that it was inadequate and that they needed additional support at work. Another source of support that they requested from the organization was extra income: "Well, as nurses at a private hospital, we expect to earn more ... The hospital director should support us more in this (extra payment)" [RN5].

\section{Discussion}

The sources of stress found in the current study were related to the three dimensions in the JDCS model. "Work context is an issue" reflected the nurses' experiences that involved the psychological demand and limited job control at the ED. The ED work characteristics, such as excessive work tasks, heavy workload, and extended work hours caused stress among the ED nurses, as has previously 
been described [1] [2] [6] and could be seen as high psychological demands [4]. Work requirements beyond nursing, e.g. performing physicians' tasks and being a mentor for new junior nurses, also caused stress. Limited decision-making authority (job control) and being in an uncontrollable situation, interfered with their capacity to complete their tasks [3] [17]. The sub-category "seeking and accepting support" reflected the nurses' experiences of inadequate support at work. When there is high demand, low control, and low support at work, a highstrain situation in the workplace can develop and lead to negative health outcomes [4]. This was observed in the category "consequences of reactions to stress" in the current study.

The ED nurses in the current study experienced patients' and their relatives' behavior as the primary stressor, due to their lack of understanding about the ED triage system, in which treatment is provided according to the severity of the patient's medical state [21]. The patients and their relatives have higher expectations and demand more from private hospitals since they pay by themselves for health care services [22]. In contrast, no disrespect from patients and their relatives were reported by the nurses at a public hospital [3], presumably since public hospital patients have different expectations and demands compared with private hospital patients. Providing clear information about waiting times and what to expect at the ED can prevent such a misunderstanding [23].

Administrative tasks regarding the patients' ability to pay also caused patientrelated stress, since the medical procedures must be the first priority in emergencies [24]. To simultaneously consider a patient's potentially serious medical condition and pay ability generates a stressing ethical dilemma. This problem does not exist at public hospitals [3]. On the other hand, the violence experienced in public hospitals was not reported at the private hospitals [3].

The ED nurses described that patients and their relatives did not trust them and only valued physicians, reflecting the view of the Thai society. Physicians have higher status [16] and nurses are viewed as simple assistants [25] [26]. This may be one reason for the negative reaction that the nurses experience from patients and their relatives.

The interviews also revealed conflicts among ED nurses, within the emergency nursing team and with other health care professionals, especially with physicians. Various levels of professional hierarchy and an unclear intra-team power distribution can cause a simple disagreement to deteriorate into serious conflict, as described by Janss et al. [26].

Occupational stress can lead to poor psychophysiological health and negative social interactions, especially with family members [1] [5]. Work-related stress reactions also contribute to incomplete and delays in nursing care [3], which can lead to impaired patient safety [27]. Some study participants in the current study considered leaving the ED because of occupational stress and job dissatisfaction. This could lead to shortage of ED nurses [28], and the shortage of nurses leads to more stress, causing a vicious circle.

Some participants coped with stressful situations in the ED by taking a break, 
making a plan and returning to solve the problem, according to Folkman and Lazarus's theory of emotion-focused and problem-focused coping [29]. In contrast to in the public hospitals [3], the ED nurses also coped with stress by turning it into a challenge, viewing it as an opportunity to demonstrate professional competence. These different reactions to stress can be described as cognitive appraisals [29]. A primary appraisal refers to the determination and assessment of events, which occur in the environment and the secondary appraisal involves an assessment of the person's ability to cope with the event. It can be interpreted as that the person only experiences stress if he or she perceives and judges the event as stressful and feels that he or she is unable to cope with it.

The ED nurses in the current study received support from other ED nurses, nurse supervisors, the ED head nurse, and the nurse manager. This support included debriefing and discussing the handling of stressful situations; the participants experienced this support as an efficient source of stress management, consistent with the findings of Healy and Tyrrell [30]. Some nurses referred to meditation and Buddhist practices as means of personal stress management and reported these methods to be effective. They could represent a difference in coping strategies between Thai and Western populations, as reported by Tyson and Pongruenphant [31]. The hospital managers provided some activities aimed at relieving stress, but the nurses perceived these to be insufficient and stated that higher salaries could be a source of work-related support. Financial rewards, as compensation for stressful work is reported to have beneficial effects, e.g. on workforce stability, occupational satisfaction and the performance of high quality work [32].

The occupational stressors experienced by the ED nurses at both types of hospitals may be related to the nature of the $\mathrm{ED}$, where nurses confront a variety of stressors that inevitably includes a heavy workload, trauma and non-trauma patients with critical conditions and other crisis situations [1] [2] [6] [7]. Consequently, nurses at both types of hospitals report a number of similar sources of stress.

\section{Methodological Considerations and Limitation}

Despite the small number of participants, the responses and the lively discussion during the interviews generated data saturation. The interviews were performed by a native Thai speaker (the first author), who also translated the transcripts into English. When direct translation was not possible, a conceptual equivalent was used. A professional linguist approved the English translations and the analyzed data in English (from codes to categories). The co-authors reviewed and checked the results by verifying the meaning and confirming the categories. However, some of the phenomena noted in this study related to the Thai culture and may not be completely transferable to a Western context.

\section{Conclusions and Implication}

The work context and situations are important sources of stress among ED 
nurses in private hospitals. In particular, patients' and their relatives' lack of understanding of the triage system led to disrespect, a stressor that is not encountered in public hospital settings. Conflict among ED nurses and other health care professionals, sometimes related to the hierarchy and power relations among health care professions, causes stress. Feelings of uncertainty about continuing working or leaving the profession were also a consequence of occupational stress among ED nurses. Hence, stress may lead to shortage of ED nurses. The working environment for ED nurses needs to be improved by, for example, developing and/or revising the regulations and policies regarding work task assigned for ED nurses, and not forcing them to perform tasks beyond nursing care, as well as creating strategies for counteracting a shortage of nurses. As the nurses in the study suggested, the organization should consider increasing the nursing staff to create a better balance between the number of patients and nurses. A better working environment, and balance between the number of patients and nurses would reduce workload and stress, encourage ED nurses to stay in the profession and ultimately maintain patient safety.

The results of this and other studies could be used to develop an instrument for measuring stress in emergency departments. Such an instrument is useful for measuring the effect of stress-reducing efforts to improve the working environment of emergency departments.

\section{Acknowledgements}

The authors gratefully acknowledge the ED nurses from the two private hospitals in Bangkok, Thailand, who participated in this study.

\section{References}

[1] Healy, S. and Tyrrell, M. (2011) Stress in Emergency Departments: Experiences of Nurses and Doctors. Emergency Nurse, 19, 31-37. https://doi.org/10.7748/en2011.07.19.4.31.c8611

[2] Adriaenssens, J., De Gucht, V., Van Der Doef, M. and Maes, S. (2011) Exploring the Burden of Emergency Care: Predictors of Stress-Health Outcomes in Emergency Nurses. Journal of Advanced Nursing, 67, 1317-1328. https://doi.org/10.1111/j.1365-2648.2010.05599.x

[3] Yuwanich, N., Sandmark, H. and Akhavan, S. (2016) Emergency Department Nurses' Experiences of Occupational Stress: A Qualitative Study from a Public Hospital in Bangkok, Thailand. Work, 53, 885-879. https://doi.org/10.3233/WOR-152181

[4] Karasek, R. and Theorell, T. (1990) Healthy Work: Stress, Productivity, and the Reconstruction of Working Life. Basic Books, New York.

[5] Sauter, S., Murphy, L., Colligan, M., Swanson, N., Hunell, J., Scharf, F., Tisdale, J., et al. (2009) Stress... at Work (NIOSH). DHHS (NIOS-)-CDC, Cincinnati.

[6] Adriaenssens, J., De Gucht, V. and Maes, S. (2015) Causes and Consequences of Occupational Stress in Emergency Nurses, a Longitudinal Study. Journal of Nursing Management, 23, 346-358. https://doi.org/10.1111/jonm.12138

[7] Adriaenssens, J., De Gucht, V. and Maes, S. (2015) Determinants and Prevalence of Burnout in Emergency Nurses: A Systematic Review of 25 Years of Research. Inter- 
national Journal of Nursing Studies, 52, 649-661.

[8] Rugless, M. and Taylor, D. (2011) Sick Leave in the Emergency Department: Staff Attitudes and the Impact of Job Designation and Psychosocial Work Conditions. Emergency Medicine Australasia, 23, 39-45. https://doi.org/10.1111/j.1742-6723.2010.01372.x

[9] WHO Western Pacific Region (2015) The Kingdom of Thailand: Health System. http://www.wpro.who.int/asia_pacific_observatory/hits/series/thailand_health_syst ems_review.pdf?ua $=1$

[10] Ramesh, M. and $\mathrm{Wu}, \mathrm{X}$. (2008) Realigning Public and Private Health Care in Southeast Asia. Pacific Review, 21, 171-187. https://doi.org/10.1080/09512740801990238

[11] Limwattananon, S., Prakongsai, P. and Tangcharoensathien, V. (2011) The Equity Impact of Universal Coverage: Health Care Finance, Catastrophic Health Expenditure, Utilization and Government Subsidies in Thailand. Consortium for Research on Equity in Health Systems (CREHS) Report.

[12] Thailand Board of Investment (2012) Thailand Medical Hub of Asia. http://www.thinkasiainvestthailand.com/download/Medical.pdf

[13] Kunaviktikul, W., Chitpakdee, B., Srisuphan, W. and Bossert, T. (2015) Preferred Choice of Work Setting among Nurses in Thailand: A Discrete Choice Experiment. Nursing \& Health Sciences, 17, 126-133. https://doi.org/10.1111/nhs.12144

[14] Yousapronpaiboon, K. and Johnson, W.C. (2013) Out-Patient Service Quality Perceptions in Private Thai Hospitals. International Journal of Business and Social Science, 4, 57-66.

[15] International Labour Organization (1985) The Professional Nursing and Midwifery Act, B.E. 2528 (1985). http://thailaws.com/law/t_laws/tlaw0294.pdf

[16] Burnard, P. and Naiyapatana, W. (2004) Culture and Communication in Thai Nursing: A Report of an Ethnographic Study. International Journal of Nursing Studies, 41, 755-765.

[17] Tyson, P.D. and Pongruengphant, R. (2004) Five-Year Follow-Up Study of Stress Among Nurses in Public and Private Hospitals in Thailand. International Journal of Nursing Studies, 41, 247-254.

[18] Raungsrijan, P. and Suppapitiporn, S. (2011) Stress of Nurses, Attitude for Development to Be a Magnet Hospital and Factors Associated with Stress of Registered Nurses in Private International Hospital. Journal of Psychiatric Association of Thailand, 56, 425-436.

[19] Helps, S. (1997) Experiences of Stress in Accident and Emergency Nurses. Accident \& Emergency Nursing, 5, 48-53.

[20] Graneheim, U.H. and Lundman, B. (2004) Qualitative Content Analysis in Nursing Research: Concepts, Procedures and Measures to Achieve Trustworthiness. Nurse Education Today, 24, 105-112.

[21] Ganley, L. and Gloster, A.S. (2011) An Overview of Triage in the Emergency Department. Nursing Standard, 26, 49-56. https://doi.org/10.7748/ns2011.11.26.12.49.c8829

[22] Zarei, A., Arab, M., Froushani, A., Rashidian, A. and Tabatabaei, S. (2012) Service Quality of Private Hospitals: The Iranian Patients' Perspective. BMC Health Services Research, 12, 1-7. https://doi.org/10.1186/1472-6963-12-31

[23] Elmqvist, C., Fridlund, B. and Ekebergh, M. (2012) On a Hidden Game Board: The Patient's First Encounter with Emergency Care at the Emergency Department. Journal of Clinical Nursing, 21, 2609-2616. 
https://doi.org/10.1111/j.1365-2702.2011.03929.x

[24] Jiménez-Herrera, M. and Axelsson, C. (2015) Some Ethical Conflicts in Emergency Care. Nursing Ethics, 22, 548-560. https://doi.org/10.1177/0969733014549880

[25] Hoeve, Y.T., Jansen, G. and Roodbol, P. (2014) The Nursing Profession: Public Image, Self-Concept and Professional Identity. A Discussion Paper. Journal of Advanced Nursing, 70, 295-309. https://doi.org/10.1111/jan.12177

[26] Janss, R., Rispens, S., Segers, M. and Jehn, K.A. (2012) What Is Happening under the Surface? Power, Conflict and the Performance of Medical Teams. Medical Education, 46, 838-849. https://doi.org/10.1111/j.1365-2923.2012.04322.x

[27] Oh, H., Uhm, D.C. and Yoon, Y.J. (2016) Workplace Bullying, Job Stress, Intent to Leave, and Nurses' Perceptions of Patient Safety in South Korean Hospitals. Nursing Research, 65, 380-388. https://doi.org/10.1097/NNR.0000000000000175

[28] Sawatzky, J.-A.V. and Enns, C.L. (2012) Exploring the Key Predictors of Retention in Emergency Nurses. Journal of Nursing Management, 20, 696-707. https://doi.org/10.1111/j.1365-2834.2012.01355.x

[29] Folkman, S. and Lazarus, R.S. (1980) An Analysis of Coping in a Middle-Aged Community Sample. Journal of Health and Social Behavior, 21, 219-239. https://doi.org/10.2307/2136617

[30] Healy, S. and Tyrrell, M. (2013) Importance of Debriefing Following Critical Incidents. Emergency Nurse, 20, 32-37. https://doi.org/10.7748/en2013.03.20.10.32.s8

[31] Tyson, P.D. and Pongruengphant, R. (2007) Buddhist and Western Perspectives on Suffering, Stress, and Coping. Journal of Religion and Health, 46, 351-357. https://doi.org/10.1007/s10943-006-9104-Z

[32] Wieck, K., Dols, J. and Northam, S. (2009) What Nurses Want: The Nurse Incentives Project. Nursing Economics, 27, 169-201.

Submit or recommend next manuscript to SCIRP and we will provide best service for you:

Accepting pre-submission inquiries through Email, Facebook, LinkedIn, Twitter, etc. A wide selection of journals (inclusive of 9 subjects, more than 200 journals)

Providing 24-hour high-quality service

User-friendly online submission system

Fair and swift peer-review system

Efficient typesetting and proofreading procedure

Display of the result of downloads and visits, as well as the number of cited articles

Maximum dissemination of your research work

Submit your manuscript at: http://papersubmission.scirp.org/

Or contact ojn@scirp.org 